published Heart Cell Atlas study. Expression was altered in DCM in all but the rarest heart cell populations. Genes identified as altered in DCM in bulk RNA-seq were compared with altered genes from each single-cell cardiac cell population. Greatest concordance between the two techniques was noted in fibroblasts, with 14 upregulated and 13 downregulated genes common across both analyses. Several of these genes were independently validated in an in vitro model of TGF $\beta$-treated human cardiac fibroblasts.

Conclusions DCM is a complex pathology involving interactions between multiple cardiac cell populations. Our analysis workflow improved resolution at the single-cell level, providing more accurate recapitulation of in vivo tissue heterogeneity. This unbiased approach has enabled the robust detection of unique disease-relevant transcriptomic alterations in specific cardiac cell populations in DCM.

Conflict of Interest None

\section{INDUCED PLURIPOTENT STEM CELL-DERIVED ENDOTHELIAL CELLS FROM HUMAN DIABETIC DONORS CARRY AN IMPRINT OF THE DIABETIC MILIEU}

\begin{abstract}
${ }^{1}$ Magdalini Eleftheriadou, ${ }^{1}$ Hojjat Naderi Meskin, 'Andrew Yacoub, 'Thomas Morrison, ${ }^{1}$ Victoria Cornelius, ${ }^{2}$ Chunbo Yang, ${ }^{3}$ Martha Gonzalez, ${ }^{1}$ Sophia Kelaini, ${ }^{1}$ Garret Carney, ${ }^{1}$ Guillermo Lopez Campos, 'Noemi Lois, ${ }^{1}$ David Grieve, ${ }^{1}$ Alan Stitt, 'Andriana Margariti. ${ }^{1}$ Queens University Belfast, Belfast, UK; ${ }^{2}$ Newcastle University; ${ }^{3}$ Cambridge University
\end{abstract}

10.1136/heartjnl-2021-BCS.9

Diabetic endotheliopathy is the main cause for impaired angiogenesis and reduced neovascularization that lead to microvascular injury and vascular complications. The pathogenic basis for vascular complications arising from diabetes is complex. Elucidation of key underlying mechanisms will help the development of novel therapies and the discovery of potential biomarkers. The ability to generate functional endothelial cells (ECs) from induced pluripotent stem cells (iPSCs) from small amounts of blood is a novel and powerful tool for cell-based therapies. Human iPSC-derived ECs (iPS-ECs) have a broad range of clinical applications including cell-based therapy, disease modelling and drug screening; they can be used in mechanistic studies towards the development of novel therapies and in the discovery of new biomarkers to be applied in regenerative medicine and treatment of diabetic vasculopathy. Here we utilize transcriptomic and proteomic technologies to assess patient-specific iPS-ECs from diabetic (DiPS-ECs) and non-diabetic (NiPS-ECS) donors $1,2,3,4$ in order to investigate the mechanisms driving endotheliopathy in diabetes. Our in vitro and in vivo models recapitulate the effects of hyperglycaemia on the vasculature in the clinical setting. RNA-seq data showed that genes and proteins involved in angiogenesis and EC function were significantly downregulated in DiPS-ECs in comparison to NiPS-ECS $(n=3, p<0.05)$. Specific epsins regulating VEGF-mediated angiogenesis were downregulated in DiPS-ECs, leading to increased signalling VEGF pathway activation6. Moreover factors involved in E-cadherin signalling, endothelial-to-mesenchymal transition and fibrosis were increased in DiPS-ECs. We detected abnormal capillary permeability and barrier integrity in DiPS-ECs using xCELLigence ${ }^{\circledR}$. DiPS-ECs had significantly reduced barrier integrity and barrier recovery $(n=3, p<0.001, \pm S E M)$ and also displayed impaired tube formation in vitro $(\mathrm{n}=3, \pm$ SEM, $\mathrm{p}<0.05)$. DiPS-ECs displayed impaired function demonstrated by decreased blood flow recovery (BFR) compared to NiPS ECs $(n=3)$ when injected to the hindlimb of mice following femoral artery ligation. Finally, our proteomic and transcriptomic analysis confirmed imbalances in several angiogenic genes including endothelial specific Roundabout protein 4 (ROBO4) that is highly involved in pathways related to angiogenesis, barrier stability and endothelial health 7. Expression of ROBO4 was found to be impaired in DiPS-ECs and transcriptomic analysis along with in vitro and in vivo studies revealed its importance in vascular development and angiogenesis. Our data support the impaired angiogenic functionality of DiPSECs cells in vitro and in vivo and show that DiPS-ECs carry an imprint of the diabetic milieu which is reflected in their dysfunction. To the best of our knowledge, we have identified a novel disease-specific signature in diabetic iPS-ECs, therefore our human iPS-EC model may serve as a valuable tool to study biological pathways and identify new treatments for diabetes-induced endotheliopathy.

Conflict of Interest None

\section{PRIMARY MITRAL REGURGITATION SUCCESSFULLY TREATED BY PERCUTANEOUS MITRAL VALVE LEAFLET REPAIR RESULTS IN POSITIVE CARDIAC REVERSE REMODELLING AND FUNCTIONAL IMPROVEMENT}

${ }^{1}$ Thomas Craven, ${ }^{1}$ Pei Gee Chew, 'Miroslawa Gorecka, 'Louise Brown, 'Arka Das, ${ }^{1}$ Amrit Chowdhary, ${ }^{1}$ Nicholas Jex, ${ }^{1}$ Sharmaine Thirunavukarasu, 'Erica Dall'Armellina, ${ }^{2}$ Eylem Levelt, ${ }^{3}$ Dominik Schlosshan, ${ }^{3}$ Christopher Malkin, ${ }^{3}$ Daniel Blackman, 'Sven Plein, ${ }^{1}$ John Greenwood. 'University of Leeds, Leeds, UK; ${ }^{2}$ University of Leeds, Multidisciplinary Cardiovascular Research Centre and Biomedical Imaging Science; ${ }^{3}$ Leeds Teaching Hospitals NHS trust

\subsection{6/heartjin-2021-BCS.10}

Background Percutaneous mitral valve leaflet repair can be an effective treatment for primary mitral regurgitation (MR) patients deemed high-risk for surgery. Accurate assessment of cardiac reverse remodelling is essential to optimise future patient selection. Cardiovascular magnetic resonance (CMR) is the reference standard for cardiac volumetric assessment and compared to transthoracic echocardiography (TTE) provides superior reproducibility in MR quantification. Prior CMR studies have analysed cardiac reverse remodelling following percutaneous intervention in combined cohorts of primary and secondary MR patients. However, as aetiology of MR can significantly impact outcomes, focused studies are warranted. Therefore, we aimed to assess cardiac reverse remodelling and quantify changes in MR following percutaneous mitral valve leaflet repair for primary MR using the reference standard (CMR).

Methods 12 patients with at least moderate-severe MR on TTE were prospectively recruited to undergo CMR imaging and 6-minute walk tests (6MWT) at baseline and 6 months following percutaneous mitral valve leaflet repair (MitraClip). CMR protocol involved: left-ventricular (LV) short axis cines (bSSFP, SENSE-2, 10mm, no gap), transaxial right-ventricular (RV) cines (bSSFP, SENSE-2, $8 \mathrm{~mm}$, no gap), two and four chamber cines and aortic through-plane phase contrast imaging, planned at the sino-tubular junction. MR was quantified indirectly using LV and aortic stroke volumes.

Results 12 patients underwent percutaneous mitral valve leaflet repair (MitraClip) for posterior mitral valve leaflet prolapse, however 1 patient declined follow up after single-leaflet clip 

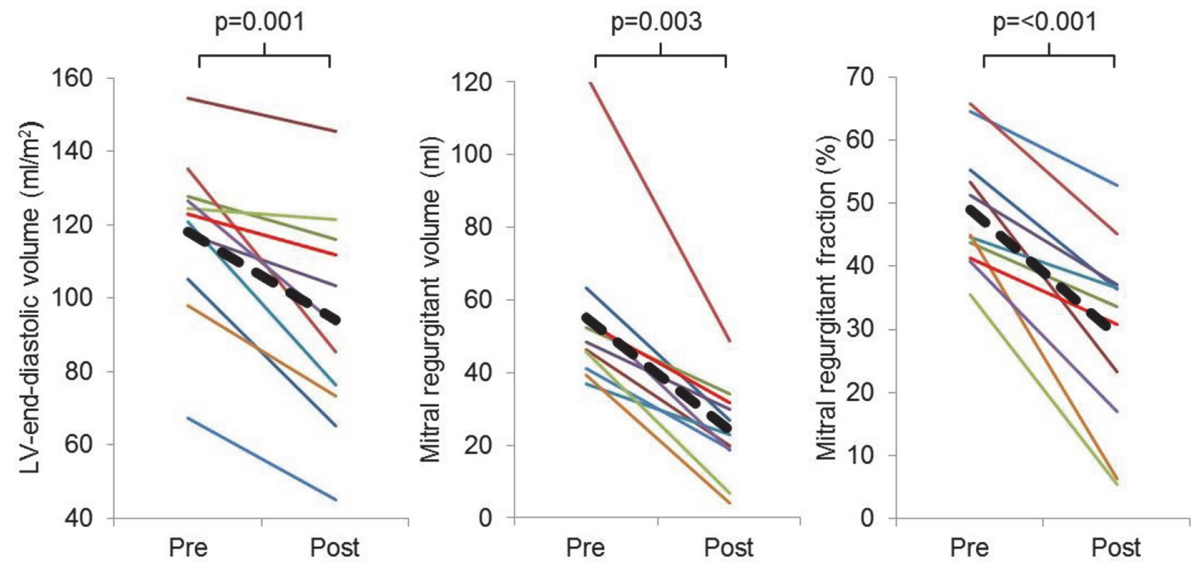

Abstract 10 Figure 1 Reduction in indexed left ventricular (LV) end-diastolic volumes and mitral regurgitation 6-months after percutaneous mitral valve leaflet repair

Dashed black line represents mean values

Abstract 10 Table 1 Functional changes6-months following percutaneous mitral valve leaflet repair

\begin{tabular}{lllll}
\hline & Baseline & Follow up & p-value \\
\hline 6-minute walk test distance (m) & & $223 \pm 71$ & $281 \pm 65$ & 0.005 \\
New York Heart Association functional class & I & 0 & 6 & 0.002 \\
& II & 3 & 4 & \\
& III & 8 & 1 & \\
IV & 0 & 0 & \\
\hline
\end{tabular}

Abstract 10 Table 2 Changes incardiac indices 6-months following percutaneous mitral valve leaflet repair

\begin{tabular}{llll}
\hline Cardiac Indices & Baseline & Follow up & p-value \\
\hline LV end-diastolic volume $\left(\mathrm{ml} / \mathrm{m}^{2}\right)$ & $118 \pm 21$ & $94 \pm 27$ & 0.001 \\
LV end-systolic volume $\left(\mathrm{ml}^{2} / \mathrm{m}^{2}\right)$ & $58 \pm 19$ & $48 \pm 21$ & 0.007 \\
LV ejection fraction $(\%)$ & $51 \pm 10$ & $51 \pm 7.8$ & 0.775 \\
Left atrial volume $\left(\mathrm{ml} / \mathrm{m}^{2}\right)$ & $108 \pm 39$ & $102 \pm 31$ & 0.565 \\
Mitral regurgitant volume $(\mathrm{ml})$ & $55 \pm 22$ & $24 \pm 12$ & 0.003 \\
Mitral regurgitant fraction $(\%)$ & $49 \pm 9.4$ & $29 \pm 14$ & $<0.001$ \\
RV end-diastolic volume $\left(\mathrm{ml} / \mathrm{m}^{2}\right)$ & $101 \pm 28$ & $103 \pm 28$ & 0.790 \\
RV end-systolic volume $\left(\mathrm{ml} / \mathrm{m}^{2}\right)$ & $58 \pm 26$ & $56 \pm 21$ & 0.477 \\
RV ejection fraction $(\%)$ & $44 \pm 8.4$ & $48 \pm 8.9$ & 0.110 \\
Right atrial area $\left(\mathrm{cm}^{2} / \mathrm{m}^{2}\right)$ & $17 \pm 4.5$ & $18 \pm 4.3$ & 0.508 \\
\hline
\end{tabular}

Abbreviations: LV, left ventricular; RV, right ventricular.

detachment resulting in 11 patients (age 83 5 years, 9 male) completing follow up imaging. At 6-months: significant improvements occurred in New York Heart Association functional class and $6 \mathrm{MWT}$ distances $(223 \pm 71 \mathrm{~m}$ to $281 \pm 65 \mathrm{~m}$, $\mathrm{p}=0.005$ ) (table 1) and significant reductions occurred in indexed left ventricular end-diastolic volumes (LVEDVi) (118 $\pm 21 \mathrm{ml} / \mathrm{m} 2$ to $94 \pm 27 \mathrm{ml} / \mathrm{m} 2, \mathrm{p}=0.001)$, indexed left ventricular end-systolic volumes $(58 \pm 19 \mathrm{ml} / \mathrm{m} 2$ to $48 \pm 21 \mathrm{ml} / \mathrm{m} 2, \mathrm{p}=0.007)$ and quantitated $\mathrm{MR}$ volume $(55 \pm 22 \mathrm{ml}$ to $24 \pm 12 \mathrm{ml}$, $\mathrm{p}=0.003)$ and $\mathrm{MR}$ fraction $(49 \pm 9.4 \%$ to $29 \pm 14 \%, \mathrm{p} \leq 0.001)$ (table 2). There were no statistically significant changes in left ventricular ejection fraction (LVEF), right ventricular dimensions/ejection fraction or bi-atrial dimensions (table 2). All patients demonstrated decreased LVEDVi and quantified MR (figure 1).

Conclusion Successful percutaneous mitral valve leaflet repair for primary $\mathrm{MR}$ results in reduction in $\mathrm{MR}$, positive $\mathrm{LV}$ reverse remodelling, preservation of LVEF, and functional improvements. Larger CMR studies are now required to further guide optimal patient selection.

Conflict of Interest Nil

\begin{tabular}{l|l}
\hline 11 & A 5 YEAR STUDY OF INFECTIVE ENDOCARDITIS \\
MANAGED BY A MULTIDISCIPLINARY TEAM IN A \\
REGIONAL CARDIOTHORACIC CENTRE: TRENDS IN \\
REFERRAL, INFECTIVE ORGANISMS AND OUTCOMES
\end{tabular}

${ }^{1}$ Rhys Wenlock, ${ }^{1}$ Eleanor Thornton, ${ }^{2}$ Sally Curtis, ${ }^{1}$ Michael Lewis, 'Luke Holland, ${ }^{1}$ Rachael James. 'Sussex Cardiac Centre, Brighton and Sussex University Hospitals NHS Trust, Brighton, UK; ${ }^{2}$ Department of Microbiology, Brighton and Sussex University Hospitals NHS Trust

\subsection{6/heartjnl-2021-BCS.11}

Introduction Infective endocarditis (IE) is associated with a protracted inpatient stay, prolonged intravenous antimicrobial therapy and significant morbidity and mortality.

Methods All patients with a diagnosis of IE at the Sussex Cardiac Centre (SCC) between 2016 and 2020 were included. Demographics, clinical characteristics, management, complications and outcomes were analysed. Logistic EuroSCORE, EuroSCORE II and Risk-E Scores were calculated for patients undergoing operative management.

Results In the 5-year period 202 patients had 209 diagnoses of IE. Seven patients had recurrent IE; 3 with the same organism but with persistent risk factors after initial treatment (Intravenous drug users [IVDUs] $\mathrm{n}=2$ and colonic polyps $\mathrm{n}=1)$. IE cases increased yearly by approximately 4 cases per year $(p<0.01)$. The increase was predominantly driven by cases from referring centres, with $41 \%$ transferred from other hospitals $(n=85)$. Oral streptococci $(n=50)$, coagulase-positive staphylococci $(n=43)$, non-oral streptococci $(n=29)$ and enterococci $(n=28)$ were the commonest organisms identified (figure 1). Only oral streptococci demonstrated a significant increase over time $(p<0.05$, figure 1$)$. Men made up $78 \%(n=163)$ of cases, with a male predominance observed in all organism groupings. Surgical 